RESEARCH PAPER RP1167

Part of Journal of Research of the National Bureau of Standards, Volume 22, January 1939

\title{
APPLICATION OF GRAPHS OF MAXIMUM USABLE FREQUENCY TO COMMUNICATION PROBLEMS
}

\author{
By Newbern Smith, Samuel S. Kirby, and Theodore R. Gilliland
}

\section{ABSTRACT}

The maximum usable frequency for any distance is the highest frequency which can be effectively used for radio sky-wave transmission over the given distance. Graphs for the maximum usable frequencies for the latitude of Washington are being published monthly. These graphs are useful in determining the best frequency for communication over a given path at a given time. The general rule is given for determining this optimum frequency: Use the highest available frequency that will not skip. This is more important in the daytime than at night, on account of absorption. The diurnal and seasonal variations recur regularly and the variations with the sunspot cycle may be estimated for a reasonable time in advance, and therefore estimations of future maximum usable frequencies may be made. The methods of applying these graphs to communication problems are discussed.

\section{CONTENTS}

Page

I. Introduction

II. Relation of vertical- to oblique-incidence transmission

III. Maximum-usable-frequency factors

IV. Maximum-usable-frequency curves

V. Choice of optimum frequency for communication

VI. Examples of use of curves

VII. Experimental check of theory

VIII. Conclusions

\section{INTRODUCTION}

Since June 1937 the National Bureau of Standards has broadcast each Wednesday, from its station WWV, values of the critical frequencies and virtual heights of the principal ionosphere layers, together with the maximum frequencies which could be used for radio skywave communication over various distances. ${ }^{1}$ Graphs ${ }^{2}$ showing the maximum usable frequencies for various distances and times of day, for average conditions during the month have been published and are being published currently. These values of maximum usable frequency are derived from the regular vertical-incidence ionosphere measurements made by the Bureau at Washington, also published currently (see footnote 2). They are representative of conditions at other places on the earth not too widely differing in latitude from that of Washington.

\footnotetext{
1 The Weekly Radio Broadcasts of the National Bureau of Standards on the Ionosphere and Radio Transmission Conditions, National Bureau of Standards Letter Circular LC499 (May 15, 1937).

${ }_{2}$ T. R. Gilliland; S. S. Kirby, N. Smith, and S. E. Reymer, Characteristics of the ionosphere and their application to radio transmission, J. Research NBS 18, 645 (1937) RP1001; Proc. Inst. Radio Engrs. 25, 823 (1937). Maximum usable frequencies for radio sky-wave transmission, 1938 to $198 \%$, J. Research NBS 20 , 627 (1938) RP1096; Proc. Inst. Radio Engrs. 26, 1347 (1938). Characteristics of the ionosphere at Washington, $D$ C., series of monthly reports beginning in the September 1937 issue of the Proc. Inst. Radio Engrs.
} 
The method of deriving maximum usable frequencies from verticalincidence ionosphere measurements has been described elsewhere. ${ }^{3}$ It is the purpose of this paper to give a brief discussion of the significance of maximum usable frequencies in communication problems and of the methods of using them.

\section{RELATION OF VERTICAL- TO OBLIQUE-INCIDENCE TRANSMISSION}

When a radio wave is sent obliquely up into an ionized region, as in transmission over a distance, the ionized medium is able to return waves of frequencies considerably higher than the vertical-incidence
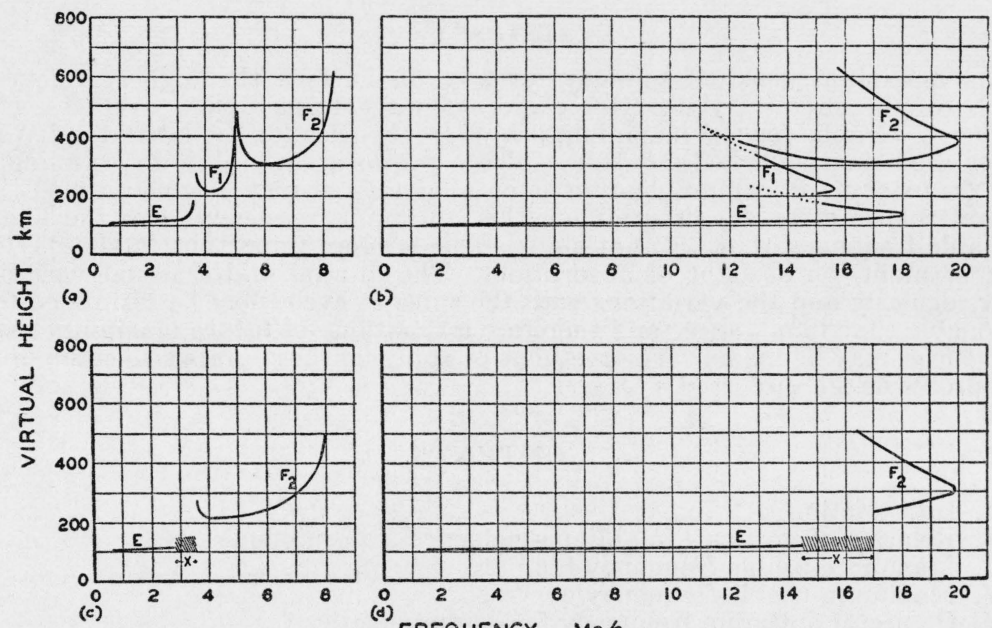

(b)

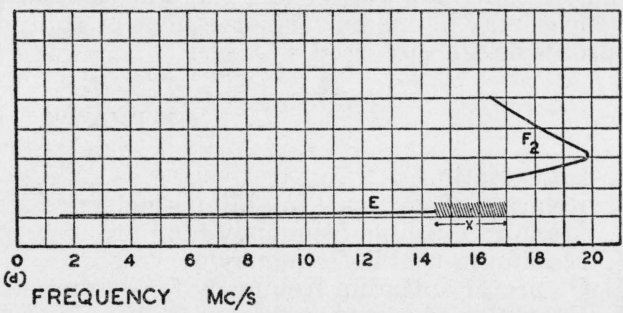

FIGURE 1.-Vertical and oblique-incidence transmission.

Curves of virtual height of ionosphere layers against frequency. Curves $a$ and $c$ are idealized vertical-incidence curves, and curves $b$ and $d$ are theoretical oblique-incidence curves derived from them for transmission over an $1,800-\mathrm{km}$ path. The dotted portions of the curves show where transmission via a given layer is shielded by reflection from a lower layer. $X$ represents a band of frequencies completely absorbed.

penetration frequency. In general, a region of given ionic density returns waves of frequencies which are higher, the greater the angle of incidence upon the layer. Thus, so far as the curvature of the earth permits, higher frequencies can be reflected, the greater the distance of transmission and the lower the height of reflection.

Figure 1 gives typical curves of virtual height against frequency, for vertical incidence, and for oblique incidence (single-hop transmission over a distance of $1,800 \mathrm{~km}$ ). In general, the virtual heights vary similarly with frequency in both vertical- and oblique-incidence cases, but the oblique-incidence curves turn back at the maximum usable frequency for each layer instead of merely curling up as they do at vertical incidence. Because of this, transmission at the maximum usable frequency of a layer is relatively unabsorbed through penetration of the layer, whereas vertical-incidence transmissions near a

3 N. Smith, The extension of normal-incidence ionosphere measurements to oblique-incidence radio transmission, J. Research NBS 19, 89 (1937) RP1013; Application of vertical-incidence ionosphere measurements to oblique-incidence radio transmission, J. Research NBS 20, 683 (1938) RP1100; The relation of radio sky-wave transmission to ionosphere measurements, Proc. Inst. Radio Engrs. (Publication pending.) 
critical frequency are usually highly absorbed. ${ }^{4}$ Because of its lower height, the E-layer may often have a greater maximum usable frequency than the $F_{1}$ or $F_{2}$ layer, although its critical frequency is usually lower.

\section{MAXIMUM-USABLE-FREQUENCY FACTORS}

It is possible to describe some of the relations between vertical- and oblique-incidence ionosphere transmission in terms of factors by which one may multiply the vertical-incidence critical frequencies to

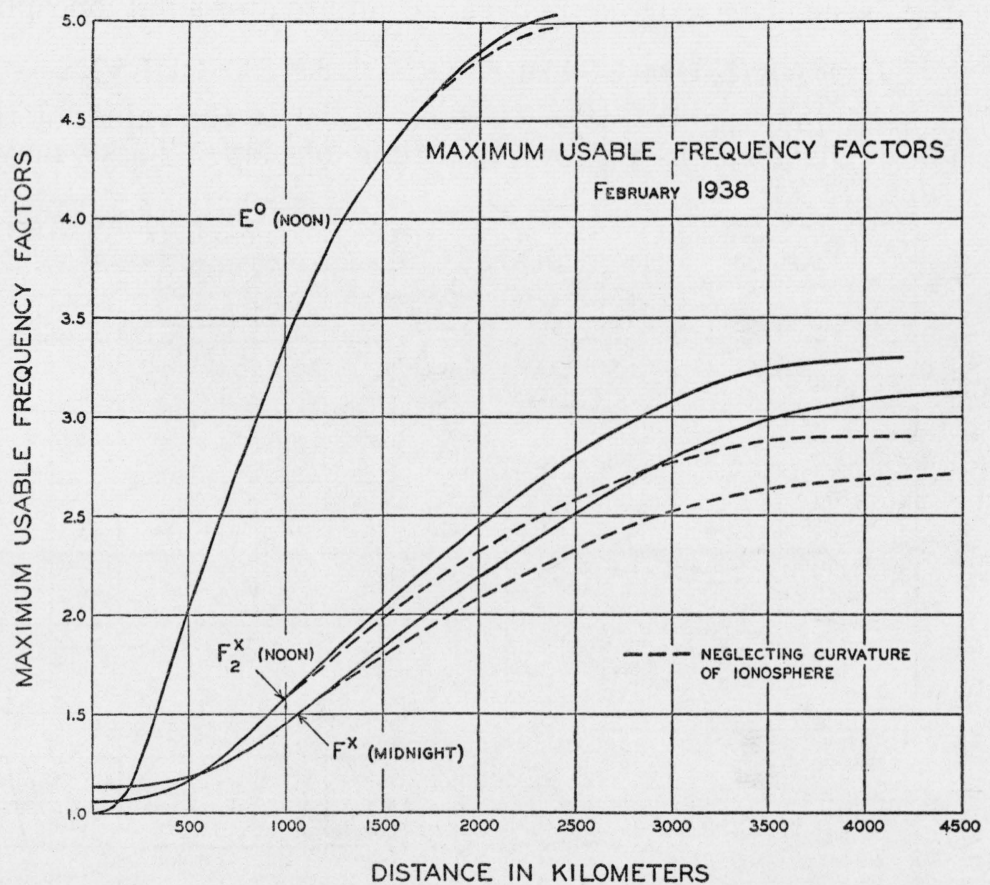

FIGURE 2.-Factors by which vertical-incidence critical frequencies (ordinary ray or "o"-component) may be multiplied in order to obtain maximum usable frequencies for transmission via the indicated layers, for February 1938.

Dashed curves are similar factors computed neglecting the curvature of the ionosphere.

get the maximum usable frequencies. It is usually most convenient to refer the factors to the critical frequency for the ordinary ray, or " 0 "-component, even though for $F$-layer transmission the " $x$ "-component has the higher maximum usable frequency. Figure 2 shows curves of typical factors by which one may multiply $f^{o}{ }_{E}, f^{o}{ }_{P_{1}}$, and $f^{o}{ }_{F_{2}}$ to get the maximum usable frequency by $E^{o_{-}}, F^{x_{1}}$, and $F^{x}{ }_{2}$-layer transmission, respectively. ${ }^{5}$ These factors were calculated for noon and midnight conditions at Washington in February 1938.

These curves indicate the order of magnitude of the values to be expected for these factors. The $E$-layer factors remain essentially the same throughout the year. The midnight $F$-layer factors are

${ }_{4}^{4}$ S. S. Kirby, L. V. Berkner, and D. M. Stuart, Studies of the ionosphere and their application to radio transmission, BS J. Research 12, 15 (1934) RP632; Proc. Inst. Radio Engrs. 22, 481 (1934).

$\checkmark$ The notation is conventional. Capital letters refer to layers, $f$ means vertical-incidence critical frequency and 0 and $x$ refer to the wave components. 
essentially the same throughout the year, but the noon $F_{2}$-layer factors are much less in summer than in winter, because of greater virtual heights in the summer. The $F$-layer factors are not unity at zero distance, because $f^{x}{ }_{p}$ is about $800 \mathrm{kc} / \mathrm{s}$ higher than $f^{0}{ }_{p}$ at Washington. The slow increase of the factors with distance at the shorter distances is due to the slow increase of sec $\phi$ with $\phi$ for small values of $\phi$. The curvature of the earth causes a slowing down of the rate of increase at the greater distances, so that the factors at these distances are not as great as if the earth were flat. The curvature of the ionosphere itself, however, partly compensates for this effect. To demonstrate this, the dashed curves in figure 2 were computed for a flat ionosphere.

\section{MAXIMUM-USABLE-FREQUENCY CURVES}

The maximum-usable-frequency data consist of the values of three variables: Distance, frequency, and time of day. These may be
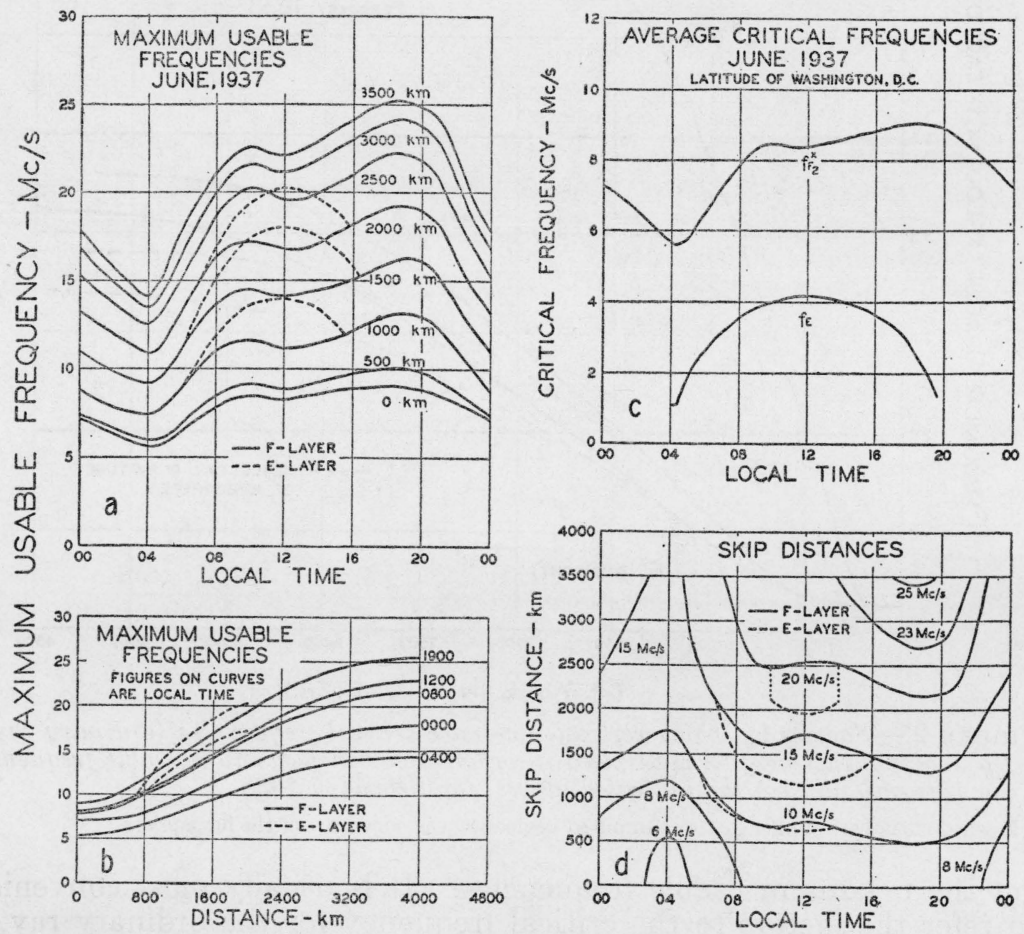

Figure 3.-Methods of plotiing maximum-usable-frequency data, June 1937

The critical-frequency curves, for vertical incidence, are also given to show their close resemblance to the maximum-usable-frequency-time curves, and to suggest the possibility of obtaining the one directly from the other.

plotted usefully in three ways, as shown in figure 3 for average conditions in June 1937:

1. Maximum usable frequency against time of day for various distances (curves $a$ ); d);

2. Skip distance against time of day for various frequencies (curves

3. Maximum usable frequency against distance for various times of day (curves $b$ ). 
Curves $c$ show the average critical frequencies at vertical incidence for various times of day during June 1937. The similarity of these curves to those in (a) permits the use of approximate factors to obtain maximum-usable-frequency curves from the average vertical-incidence curves for a given month.

Of these three ways of plotting the data, that using the curves of maximum usable frequency against distance is perhaps the least useful, since neither the maximum usable frequencies nor the skip distances vary monotonically with time of day. This type of curve is of interest chiefly where the data are limited, for example, where the maximum-usable-frequency data are available for but one or two definite times, such as noon and midnight. It indicates merely the maximum usable frequencies and the skip distances to be expected for conditions similar to those for which the curves are plotted. For example, where the curve is given for noon, the values may be used as typical for as many hours as those during which the ionosphere conditions resemble noon conditions.

The curves of maximum usable frequency against time of day are the ones published monthly in the Proceedings of the Institute of Radio Engineers by the National Bureau of Standards, and contain the available data most concisely and completely, and in a form which can be readily used. This type of curve is particularly adapted to the service of fixed radio stations, where the distance of transmission is fixed and certain frequencies are available, and it is desired to determine the optimum hours to use certain frequencies or the optimum frequencies to use during certain hours.

The skip distance-time curves present the same data as those given in the maximum-usable-frequency-time curves, but in a form not as well adapted to easy interpolation. This type of curve is particularly adapted to a mobile radio service, where the distance varies continuously, and it is desired to determine the optimum frequency to use in communication with a mobile unit at a given time. This of course applies to distances great enough to necessitate sky-wave communication.

The maximum-usable-frequency curves represent only the first step in the solution of the problem, which is the choice of suitable frequencies for a communication service. The next step consists in determining the maximum usable frequency for a given time and over a specific transmission path.

The geographical part of the ionosphere which controls long-distance high-frequency radio transmission is that part traversed by the wave in passing from the transmitter to the receiver. In the case of single-hop transmission, this part is half-way between the transmitter and the receiver. For places not differing greatly in latitude, ionosphere conditions are essentially the same at the same local time. For places differing greatly in latitude, ionosphere data for the several latitudes should be used if available.

On days when the ionosphere is undisturbed the maximum usable frequency at a given hour may be considered to vary but little (usually not over $\pm 15 \%$ ) from the average for the month. On days of ionosphere storms (disturbances of the ionosphere of the type associated with magnetic storms), ${ }^{6}$ however, variations are often greater than 50 percent from the average.

- S. S. Kirby, T. R. Gilliland, E. B. Judson, and N. Smith, The ionosphere, sunspots, and magnetic storms, Phys. Rev. 48,849 (1935).

S. S. Kirby, T. R. Gilliland, N. Smith, and S. E. Reymer, The ionosphere, solar eclipse, and magnetic storm, Phys. Rev. 50, 258 (1936). 
The maximum theoretically possible distance range for single-hop transmission, corresponding to zero angle of departure above the horizontal, is about $2,400 \mathrm{~km}$ for $E$-layer transmission, and about 3,500 to $4,500 \mathrm{~km}$ for $F_{2}$-layer transmission, depending on the virtual height of the layer. Practically, it is usually impossible to realize high-frequency communication at this zero angle of departure over land because of absorption at the ground. Observations of transmissions within the continental United States have indicated that angles

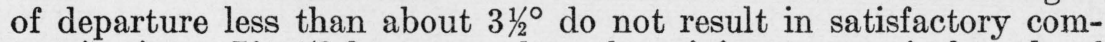
munication. If $3 y^{\circ}$ be assumed as the minimum practical angle of departure over land, the maximum distance for single-hop transmission is about $1,700 \mathrm{~km}$ for $E$ - and 3,000 to $3,500 \mathrm{~km}$ for $F_{2}$-layer transmission. Single-hop transmission may often be possible at greater distances, while at the same time multihop transmission may be more efficient.

In determining the maximum usable frequencies for a given time and transmission path, it is necessary to ascertain the number of hops and the type of transmission to be expected over the path, and the local time at the midpoint of each hop. The length of each hop depends on the virtual height at the midpoint; the higher the layer is, the longer the hop. The height, in turn, depends on the frequency; the closer this is to the maximum usable frequency, the greater the virtual height. Table 1 gives approximately the heights in kilometers which may be used in estimating the number and length of hops for frequencies at, 20 percent below, or 40 percent below the maximum usable frequency (abbreviated muf). Since the maximum usable frequency is a function of the length of hop, it is necessary to carry out the calculation by a method of approximation. The existence of paths of different lengths for multihop transmission by the same layer may be important, for one of the hops may involve reflection near the maximum usable frequency, and another hop may involve reflection considerably below it.

TABLE 1.-Virtual heights to be used in estimating the number and length of hops for transmission via different layers

\begin{tabular}{|c|c|c|c|}
\hline \multirow[b]{2}{*}{ Layer } & \multicolumn{3}{|c|}{ Frequencies } \\
\hline & at muf & $\begin{array}{c}20 \% \text { below } \\
\text { muf }\end{array}$ & $\begin{array}{l}40 \% \text { below } \\
\text { muf }\end{array}$ \\
\hline $\begin{array}{l}\text { Night } F \\
\text { Summer day } F_{2}\end{array}$ & $k m \quad \begin{array}{l}420 \\
450\end{array}$ & $\mathrm{~km}_{350}$ & $\begin{aligned} k m & \\
& \end{aligned}$ \\
\hline $\begin{array}{l}\text { Summer day } F_{1-} \\
\text { Winter day } F_{7}\end{array}$ & $\begin{array}{l}450 \\
220\end{array}$ & $\begin{array}{l}380 \\
260\end{array}$ & $(-2)$ \\
\hline${ }_{E}$ inter day $F_{2}$ & $\begin{array}{l}350 \\
130\end{array}$ & $\begin{array}{l}300 \\
115\end{array}$ & $\begin{array}{l}250 \\
110\end{array}$ \\
\hline
\end{tabular}

\section{CHOICE OF OPTIMUM FREQUENCY FOR COMMUNICATION}

The process has been outlined for determining the maximum usable frequency for the given path and time, but it yet remains to select the optimum frequency for communication from the available frequency spectrum below the maximum usable frequency. We may state a simple rule for this, that is, choose the highest available frequency that will not skip, allowing for variations in critical frequency. The varia- 
tions of critical frequencies and maximum usable frequencies below the monthly average for a given hour are usually less than 15 percent on the quiet days; therefore we should ordinarily choose a frequency at least 15 percent below the average maximum usable frequency to avoid the danger of skipping. As the frequency is lowered, the absorption of the waves is increased, especially in the daytime. As the absorption increases, the efficiency of the transmission decreases, until conditions may be reached where enormous power is necessary.

In general, however, fair efficiency of communication may be provided in the daytime by frequencies somewhat greater than half the maximum usable frequencies, and at night by frequencies down to somewhat less than half the maximum usable frequencies. Definite limits cannot be set because: (1) there is no sharp dividing line between frequencies at which absorption is high and those at which it is low, and (2) there are large irregular variations of absorption with time over both short and long periods.

At frequencies near the maximum usable frequencies there is relatively little difference between night and day absorption. As the frequency is lowered, however, the daytime absorption increases relatively much more rapidly.

\section{EXAMPLES OF USE OF CURVES}

A few examples of the use of the graphs will now be given. First, let us take an example of the use of the skip-distance-time graphs in a mobile radio service. Figure 4 shows a hypothetical airplane trip
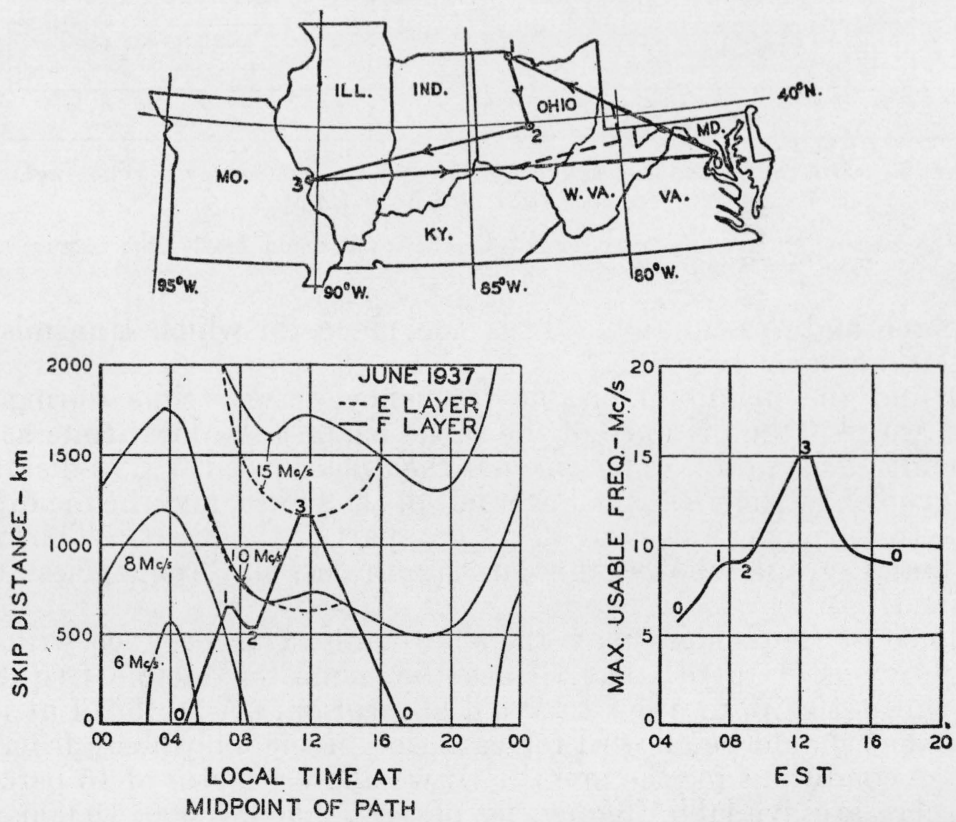

FigURE 4.-Determination of frequencies for hypothetical airplane trip from Washington, D. C. (0), to Toledo, Ohio (1), to Columbus, Ohio (2), to St Louis, Mo. (3), and back to Washington.

Lower left eurve is a plot of the midpoint of the path from Washington to the airplane against local time at this midpoint. Curve at lower right is maximum usable frequency for this trip against time at Wash. ington. 
in June 1937. The plane is supposed to leave Washington at 0500 EST, flying at 150 miles per hour, and fly to Toledo, Ohio, remaining there 20 minutes; thence to Columbus, Ohio, remaining there 30 minutes; thence to St. Louis, Mo., remaining there 45 minutes, and thence back to Washington at 1720 EST. The heavy dashed line on the map shows the course of the midpoint of the distance from Washington to the plane. This is the place where a radio wave must be reflected from the ionosphere for communication between
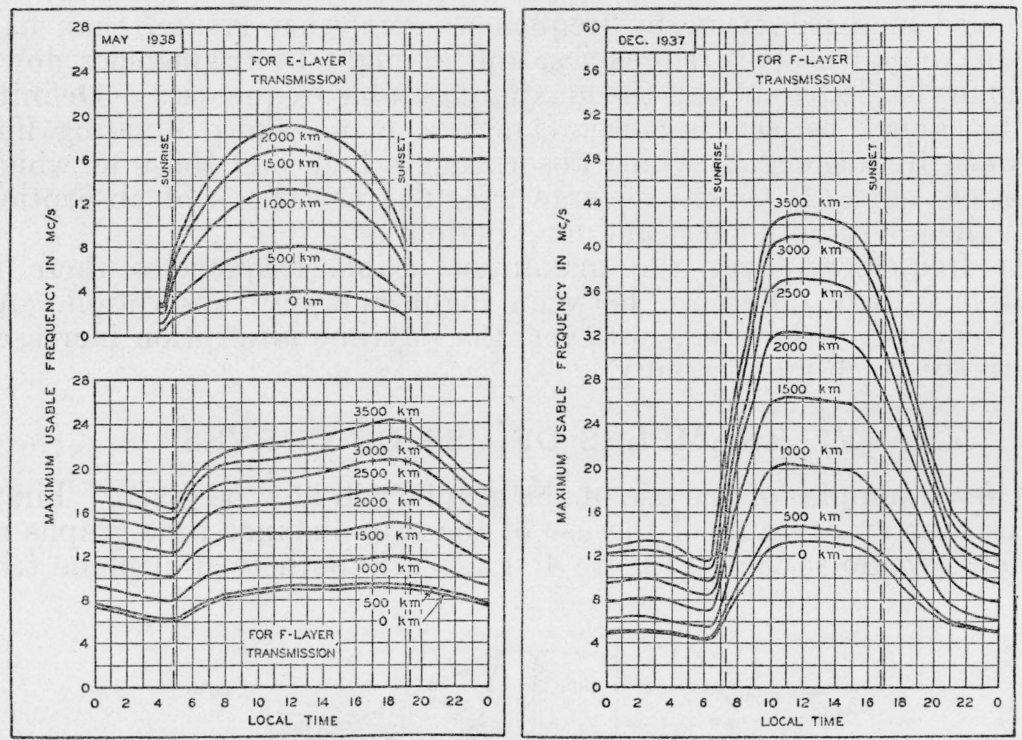

FIGURE 5.-Maximum-usable-frequency-time curves obtained at Washington for December 1937 and May 1938.

No $E$-layer curves are given for December 1937 , because the maximum usable frequencies for $E$-layer were far below those for $F$-layer transmission.

the plane and Washington. It is the place for which transmission calculations must be made.

To find the maximum usable frequency at any time during the flight, we plot the distance of the plane against the local time at the midpoint of the path. This curve is then placed on the skip-distancetime graphs, as shown at the lower left of the figure, and the maximum usable frequency can be read off for any part of the flight. The lower right-hand graph shows the maximum usable frequencies thus obtained.

Choice of frequencies may then be made in accordance with the rule previously stated, that is, use the highest available frequency that will not skip, in order to avoid absorption. A factor of at least 15 percent should be allowed for variation of the ionosphere from the average conditions for the month. If we allow a factor of 15 percent, and there are available frequencies of, say, 2,500, 5,000, 10,000, and $14,000 \mathrm{kc} / \mathrm{s}$, then: $5,000 \mathrm{kc} / \mathrm{s}$ should be used from 0500 to $1000 \mathrm{EST}$ and from 1400 to $1720 \mathrm{EST} ; 10,000 \mathrm{kc} / \mathrm{s}$ should be used from 1000 to $1400 \mathrm{EST} ; 2,500 \mathrm{kc} / \mathrm{s}$ is too low for daytime use and would be highly absorbed; and 14,000 kc/s is too near the maximum usable frequency and might skip. 
As an example of the use of the maximum-usable-frequency-time curves in a fixed service, let us take the cases of single-hop transmission from Washington to Chicago, Ill., a distance of $1,000 \mathrm{~km}$, and of two-hop transmission from Washington to San Francisco, Calif., a distance of $4,000 \mathrm{~km}$. Figure 5 shows maximum-usable-frequencytime curves for December 1937 and May 1938, representative of winter and summer conditions at Washington at the present phase of the sunspot cycle. In the winter there are no E-layer curves given, for

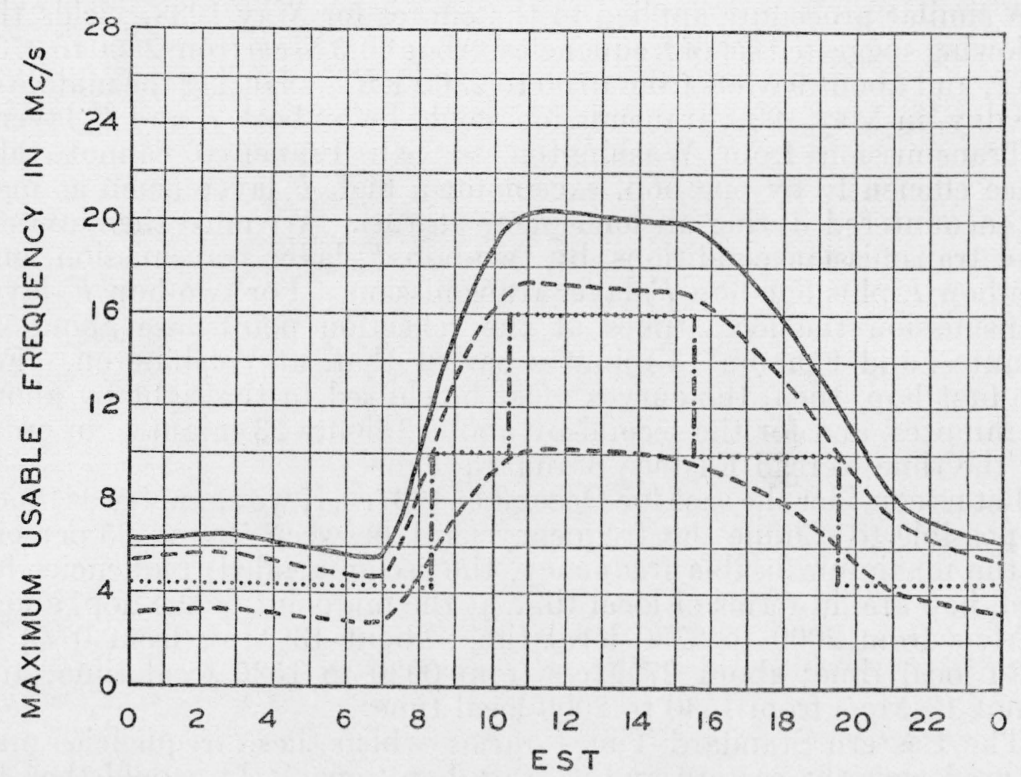

FigURE 6.-Graph showing method of determining frequencies to be used over a 1,000-km path in December $193 \%$.

Dotted lines show frequencies to be used and vertical dot-dashed lines show times for changing frequency.

then the $F_{2}$ layer determines the maximum usable frequency in the daytime. In the summer, however, the E-layer is the determining factor at some distances.

For single-hop transmission to Chicago the local time at the reflection point is about 22 minutes earlier than at Washington. Washington time is 8 minutes earlier than Eastern Standard Time. The maximum-usable-frequency curve, which reads local time at the midpoint of the path, must be displaced about 30 minutes to the right on the coordinate paper so that the time will be Eastern Standard Time. We shall allow a 15 percent minimum factor of safety to avoid skipping, and avoid as much as possible a case where the frequency employed is 50 percent or more below the maximum usable frequency.

A suggested method for determining the frequencies to be used is as follows. The maximum-usable-frequency curve for $1,000 \mathrm{~km}$ should be replotted and displaced as outlined above so that the time is Eastern Standard Time. The resulting curve is shown in figure 6, for December 1937. Two auxiliary curves are drawn on the same graph, one 15 percent below and the other 50 percent below the maximum-usable-frequency curve. These are the dashed curves in figure 6 . The frequencies chosen should then lie between these two 
auxiliary curves and should be chosen so as to involve as few changes of frequency as possible during the day. The dotted lines show one possible set of frequencies which might be used over this path during this month. These frequencies are: About $4.5 \mathrm{Mc} / \mathrm{s}$ from 1930 to $0830 \mathrm{EST}$; about $10 \mathrm{Mc} / \mathrm{s}$ from 0830 to $1030 \mathrm{EST}$; about $16 \mathrm{Mc} / \mathrm{s}$ from 1030 to $1530 \mathrm{EST}$; and about $10 \mathrm{Mc} / \mathrm{s}$ from 1530 to $1930 \mathrm{EST}$. The vertical dot-dashed lines show the times at which frequency should be changed.

A similar procedure applied to the curves for May 1938, yields the following suggested set of frequencies: About 6.5 Mc/s from 2300 to 0700 EST, and about $9 \mathrm{Mc} / \mathrm{s}$ from 0700 to $2300 \mathrm{EST}$. During the middle of the day, in May 1938, transmission would be by both $E$ and $F_{2}$ layers.

Transmission from Washington to San Francisco cannot take place efficiently by one hop, except for a high $F$ layer (such as may be encountered during an ionosphere storm). We must then investigate transmission conditions by two-hop $F_{2}$-layer transmission, and one-hop $E$ plus one-hop $F_{2}$-layer transmission. For two-hop $F_{2}$-layer transmission the local times at the reflection points are about 45 minutes, and 2 hours 15 minutes earlier than at Washington. For the first hop, then, the curves must be shifted to the right by about 53 minutes, and for the second by about 2 hours 23 minutes, in order for the time to read Eastern Standard Time.

Let us consider the case for December 1937. If we again try as much as possible to confine the frequency used between 50 and 85 percent of the maximum usable frequency, the recommended frequencies for each hop are, in terms of local time at the midpoint of the hop, about $6 \mathrm{Mc} / \mathrm{s}$ from 2000 to 0730 local time; about $13 \mathrm{Mc} / \mathrm{s}$ from 0730 to 0930 local time; about $27 \mathrm{Mc} / \mathrm{s}$ from 0930 to 1530 local time; and about $13 \mathrm{Mc} / \mathrm{s}$ from 1530 to 2000 local time.

The Eastern Standard Times during which these frequencies may be used over the eastern and western hop, separately, would then be (a) for eastern hop: $6 \mathrm{Mc} / \mathrm{s}$ from 2053 to $0823 \mathrm{EST} ; 13 \mathrm{Mc} / \mathrm{s}$ from 0823 to $1023 \mathrm{EST} ; 27 \mathrm{Mc} / \mathrm{s}$ from 1023 to $1623 \mathrm{EST}$; and $13 \mathrm{Mc} / \mathrm{s}$ from 1623 to $2053 \mathrm{EST}$; and (b) for western hop: $6 \mathrm{Mc} / \mathrm{s}$ from 2223 to $0953 \mathrm{EST} ; 13 \mathrm{Mc} / \mathrm{s}$ from 0953 to $1153 \mathrm{EST} ; 27 \mathrm{Mc} / \mathrm{s}$ from 1153 to $1753 \mathrm{EST}$; and $13 \mathrm{Mc} / \mathrm{s}$ from 1753 to $2223 \mathrm{EST}$.

The maximum usable frequency for the entire path is the lower of the maximum usable frequencies over the two hops. If we combine the results for the two hops, then the recommended frequencies are: About $6 \mathrm{Mc} / \mathrm{s}$ from 2053 to $0953 \mathrm{EST}$; about $13 \mathrm{Mc} / \mathrm{s}$ from 0953 to $1153 \mathrm{EST}$; about $27 \mathrm{Mc} / \mathrm{s}$ from 1153 to $1623 \mathrm{EST}$; and about $13 \mathrm{Mc} / \mathrm{s}$ from 1623 to $2053 \mathrm{EST}$.

At certain times of day the absorption over one of the hops can be avoided only by the use of a frequency greater than the maximum usable frequency over the other hop. These times are thus particularly unfavorable for consistent communication over this path. This condition will be most likely to occur between about 0823 and 1153 EST (absorption being greater on the eastern hop) and between about 1623 and 2223 (absorption being greater on the western hop).

The other possible path, by one-hop $E$ plus one-hop $F$-layer transmission, must be considered during the daytime. By the geometry of the path the length of the $E$ hop would be about $1,200 \mathrm{~km}$, and the maximum usable frequency would be far below that for $F$-layer transmission over the same path. The calculation for two-hop $F$-layer transmission will give the best frequencies to use. 
The calculation for two-hop E-layer transmission may be made in the same manner for May 1938. For one-hop $E$ plus one-hop $F$ layer transmission the length of the $E$ hop could be only about 800 $\mathrm{km}$, so the two-hop $F$-layer transmission would be the preferred one here also.

One other point should be noted. If the local time at the transmitter is on the up- or downgoing parts of the maximum-usablefrequency curve for a given distance, then a different frequency should be used for transmitting over that distance eastward from the transmitter than over the same distance westward from the same transmitter. This does not mean that different frequencies should be used in transmitting in opposite directions over the same path. Except in unusual cases, transmission is essentially the same in the two directions over the same path.

\section{EXPERIMENTAL CHECK OF THEORY}

Figure 7 shows an example of the type of experimental confirmation of the maximum-usable-frequency theory that has been obtained. The upper part of the figure is a continuous record by the National

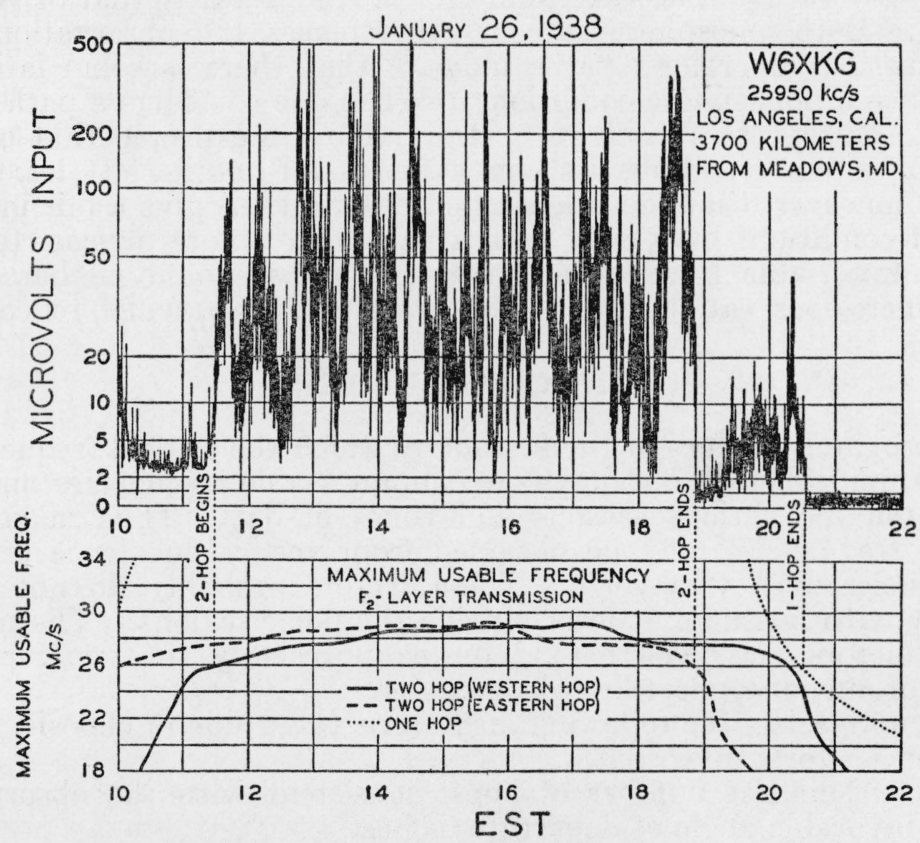

FIGURE 7.-Field-intensity record of transmission from $W 6 X K G$.

Below are given the maximum-usable-frequency curves computed from ionosphere data taken at Washington on the same day. The major changes in transmission coincide with the times when the maximum usable frequency passes through $26 \mathrm{Mc} / \mathrm{s}$. (The intensity around $1100 \mathrm{EST}$ is really nearly zero; the discrepancy is due to a drift in the zero of the recorder).

Bureau of Standards of the field intensity of W6XKG, Los Angeles, Calif., on $25,950 \mathrm{kc} / \mathrm{s}$, at a distance of $3,700 \mathrm{~km}$. In the lower part are shown graphs of the calculated maximum usable frequency over this distance, for both one- and two-hop transmission. This distance is slightly beyond the maximum distance for good one-hop transmission during the winter day, on the basis of the $312^{\circ}$ angle of depar- 
ture. The changes between one- and two-hop transmission are well marked, and the ratio of signal strength is well over 100:1. This difference is due partly to the increased absorption over the flatter trajectory and partly to the unfavorable angle of departure. It should be noted how the times of beginning and ending of two-hop transmission agree with the times the calculated maximum usable frequency passed through about $26 \mathrm{Mc} / \mathrm{s}$ at the western and eastern hops, respectively. The two-hop transmission began as soon as the western hop permitted and ended when the eastern hop failed.

After the failure of two-hop transmission, the station still came in by one hop. As the evening progressed the intensity increased, owing to the departure of the daytime absorption and the rise in the height of the layer, with a consequent more favorable angle of departure. The failure of single-hop transmission also is seen to agree with the fall of the calculated maximum usable frequency through $26 \mathrm{Mc} / \mathrm{s}$.

The National Bureau of Standards has checked its calculation of maximum usable frequencies against many records such as this, and it is believed that, for the ionosphere conditions on which they are based, the error is inappreciable for practical purposes. They are assumed to be good, however, only for latitudes nearly that of Washington. Both ionosphere data at other places and observations on transmissions over long paths indicate that there is some latitude difference in ionosphere conditions. When one considers a path such as from Europe to Washington, it is not to be expected that extrapolation would yield results consistent in all details. It has been found, however, that the Washington observations give a fair indication of conditions over even such a path. For more precise results, maximum usable frequencies over such paths should be based on ionosphere observations at latitudes closer to those at which reflections occur.

\section{CONCLUSIONS}

Approximate values of the factors by which the critical frequencies can be multiplied to obtain the maximum usable frequencies may be tabulated for various seasons and times of day. Thus maximum usable frequencies may be obtained from vertical-incidence critical frequencies, and vice versa. These factors probably do not vary greatly with latitude, at least outside the polar regions. The use of these factors may well extend quite appreciably the geographical range of our ionosphere knowledge.

To summarize, the following may serve as a guide in choosing best frequencies for a given path.

1. The smallest number of hops, consistent with the absorption and a favorable angle of departure, is best.

2. The highest frequency, up to within a variation factor (usually about $15 \%$ ) of the maximum usable frequency, is best.

3 . The lower the frequency the greater the absorption and the less the efficiency of transmission.

And finally, the use of the maximum-usable-frequency factors, in conjunction with our present knowledge of ionosphere conditions, suggests the possibility of estimating usable frequencies months and perhaps even years in advance, in accordance with the variations of season and of the sunspot cycle.

Washington, August 22, 1938. 